\title{
Intraventricular conduction defects in acute myocardial infarction. What is the influence of primary coronary angioplasty on their prognosis?
}

\author{
Álvaro DB Bordalo*, Armando L Bordalo-Sá, Maria José Correia, Carlos Ribeiro and A Nunes Diogo \\ Cardiac Intensive Care Unit, Cardiology Department, Hospital de Santa Maria, University of Lisbon School of Medicine, Lisbon, Portugal
}

\begin{abstract}
Introduction and objective: In the pre-thrombolytic era, patients (pts) with a major intraventricular conduction defect (IVCD) - right bundle branch block (RBBB), left bundle branch block (LBBB), left posterior hemiblock (LPH), advanced (QRS > $105 \mathrm{msec}$ ) left anterior hemiblock (LAH) or left IVCD with normal axis and QRS > 115 msec - acquired during ST-segment elevation myocardial infarction (STEMI) very frequently had a poor in-hospital prognosis. The objective of this study is to evaluate, in an era characterized by the prominent use of emergent mechanical coronary reperfusion in STEMI, what is the impact of emergent coronary angioplasty (CA) on the prognosis of pts with STEMI complicated by IVCD.
\end{abstract}

Methods: Retrospective observational study of 605 STEMI pts consecutively admitted between January 1st, 2008 and June 30, 2012, with a mean admission time of $174 \mathrm{~min}$, and referred to emergent coronary angiography with the intention to perform primary (effective in 92\% pts, $308 \pm 343$ min after STEMI clinical start) or rescue (effective in 4\% pts) CA - group A. All 12-lead ECG tracings recorded since admission to discharge/decease were reviewed and screened for the presence of LAH, advanced LAH, LPH, RBBB (isolated or associated with left IVCD), LBBB, aspecific left IVCD and aspecific bilateral IVCD; the clinical profile and in-hospital evolution of IVCD pts were analyzed. Pts with new RBBB $(n=40)$ were matched with control IVCD-free pts $(n=40)$ for gender, age, clinical and angiographic STEMI characteristics, and comorbidities. Some data from the present study were compared with the results of two other studies from our Group performed in the pre-thrombolytic era: a) group B - 474 pts with acute myocardial infarction (AMI), consecutively admitted in 1980-1982 (mean age of 62 and inhospital mortality of 22\%); b) group C - 764 AMI pts consecutively admitted up to 3 hours of AMI evolution in 1982-1989 (mean age of 59 and 30-day mortality of $8 \%$ in arrhythmia-free pts).

Results: 1) Prevalences (group A): preexistent IVCD - 14.5\%; acquired IVCD - 27.6\% (transient in 52\% pts, sustained in 48\%) and, specifically, isolated LAH $15.0 \%$ (advanced LAH - 1.8\%), isolated LPH - 1.3\%, isolated RBBB - 3.3\%, RBBB+LAH - 3.3\%, RBBB+LPH - 0.2\%, aspecific left IVCD - 2.3\%. 2) Pts with new RBBB vs controls: ejection fraction $<35 \%-40.0 \%$ vs $12.5 \%$ ( $<<0.025$ ); extremely severe lesion of the infarct-related artery $-50.0 \%$ vs $22.5 \%$ ( $<<0.025)$. 3 ) Inhospital mortality (group A): preexistent IVCD - 6.9\%; IVCD-free pts - 5.1\% (5.0\% in RBBB-matched controls); acquired IVCD (global value) - 13.2\%; acquired major IVCD - 26.9\% (vs 62\% in group C; $\mathrm{p}<0.001$ ), and specifically, $\mathrm{LBBB}-35.7 \%$ (vs $46.7 \%$ in group $\mathrm{B}$; $\mathrm{p}=0.248$ ), sustained isolated RBBB - $40.0 \%$ (vs $20.0 \%$ in transient RBBB), RBBB+LAH - 45.0\%; acquired/indetermined RBBB (global value) - 37.2\% (vs 58\% in group B; p>0.1); $95 \%$ mortality in pts with acquired IVCD is due to major IVCD cases (mostly pts with RBBB or LBBB).

Conclusions: In the era of STEMI mechanical reperfusion, the presence of preexistent IVCD has no prognostic influence. Emergent CA significantly improved the global prognosis of acquired major IVCD but did not significantly modify the ominous prognosis of pts with STEMI complicated by LBBB or sustained RBBB, either isolated or associated with LAH; to improve the poor prognosis in these cases, primary CA should be performed within the first 2 hours of STEMI evolution.

\section{Introduction}

Occurrence of an intraventricular block (IVB) in patients with acute myocardial infarction (AMI) is a relatively frequent event. Prior to the introduction of coronary reperfusion therapies (initially the fibrinolytic treatment, and later, the mechanical reperfusion), the patients showing major IVB - that is, incomplete or complete right bundle branch block (RBBB), non-brady dependent complete left bundle branch block (LBBB) pattern, left posterior hemiblock (LPH), advanced (QRS duration > $105 \mathrm{msec}$ ) left anterior hemiblock (LAH), and left IVB with normal axis, non-LBBB pattern but a QRS duration of $120 \mathrm{msec}$ or longer - acquired through the acute phase of myocardial infarction, frequently had a poor or very poor prognosis, especially in patients presenting with RBBB [1,2] or complete LBBB [2]. Later, in the thrombolytic era, patients with ST elevation AMI (STEMI) complicated by RBBB or complete LBBB still maintained a short-term prognosis significantly worse than the individuals without acute bundle branch block $[3,4]$. On the other hand, the first reports on this subject from the mechanical reperfusion era persistently revealed a significantly higher short-term mortality in STEMI patients with acute RBBB or complete LBBB [5-7].

The purpose of this work - in a time when the prominent role of primary coronary angioplasty (CA) as the gold standard for STEMI

Correspondence to: Álvaro DB Bordalo, Cardiac Intensive Care Unit, Cardiology Department, Hospital de Santa Maria, University of Lisbon, School of Medicine, Lisbon, Portugal, Tel: +351 21798 5100, E-mail: alvarodbb@hotmail.com

Key words: Intraventricular conduction defect, acute myocardial infarction, right bundle branch block, left bundle branch block, left anterior hemiblock, primary coronary angioplasty, prognosis

Received: January 10, 2018; Accepted: January 28, 2018; Published: January 31, 2018 
treatment is confirmed - is to re-assess the impact of coronary angioplasty on the in-hospital prognosis of patients with acquired IVB complicating STEMI, evaluated in the 'real world' practice.

\section{Methods}

This is a retrospective observational study of 605 patients with STEMI consecutively admitted in our Cardiac Intensive Care Unit between January $1^{\text {st }}, 2008$, and June 30,2012 , and submitted to emergent coronary angiography with the intention to perform primary (in most cases) or rescue CA (in cases of failed thrombolysis performed in another institution). The mean age of patients was $62+-13$ years, and they had had initial medical attendance (in ours or in another institution) within a mean of 174 minutes over the apparent clinical initiation of STEMI. Twenty-seven per cent were women, 38 per cent had diabetes, 13 per cent showed evidence of prior myocardial infarction, and 11 per cent exhibited chronic angina pectoris or had prior unstable angina. Primary CA was performed in 92 per cent of patients (within a mean of $308+-343$ minutes after the start of symptoms), and rescue CA in four per cent of patients; a coronary intervention was excluded in four per cent of subjects for diverse reasons.

Coronary angioplasty characteristics (in all patients who underwent coronary intervention): coronary stenting (bare metal or drug-eluting stents) in 91 per cent of patients, thrombectomy in 34 per cent of cases, double (aspirin plus clopidogrel) antiaggregant platelet therapy in all individuals, and adjuvant GPIIb/IIIa-inhibiting treatment in 49 per cent of patients. As additional measures, intraaortic balloon pumping, intensive inotropic drug support, intracoronary nitrates administration, temporary pacing, and mechanical ventilation support were used in, respectively, 4.1 per cent, 5.1 per cent, 3.5 per cent, 2.8 per cent, and 3.3 per cent of patients. CA was not successful in one per cent of subjects. Persistent (partial or severe) no-reflow was seen in 7.6 per cent of patients, and the acute stent thrombosis rate was 0.5 per cent.

All electrocardiographic (ECG) tracings (12 leads or more), usually recorded daily (but multiple ECG recordings could be obtained during a 24-hour period as needed) since the admission until the hospital discharge or death occurred, were carefully reviewed. Several IVB types - LAH, advanced LAH, LPH, RBBB (either incomplete or complete, either isolated or left IVB-associated), complete LBBB, aspecific left IVB, and bilateral IVB - were searched, and their diagnosis was based on previously defined ECG criteria $[8,9]$.

The clinical profiles of patients with an IVB diagnosis were evaluated, and their in-hospital evolution reviewed.

A matching of patients showing acutely acquired RBBB $(n=40)$ was a posteriori made with a sample $(n=40)$ of selected patients without preexistent or acquired IVB, in terms of gender, age, presence of a large STEMI (compromising more than five echocardiographic segments of the left ventricle), evidence of prior infarct, diabetes and other significant comorbidities (chronic kidney disease, multiple territory vascular disease, chronic obstructive lung disease, obesity), and AMI culprit artery characteristics (left main coronary artery/left anterior descending artery vs right coronary artery/circumflex artery).

The 605 patients proposed to emergent coronary revascularisation constituted the group A.

Several data concerning the group A patients were subsequently compared with the results obtained in two prior studies performed in our Department in the pre-thrombolytic era [2,10]: 1) a study involving 474 AMI patients consecutively admitted in 1980-1982 (with a mean age of 62 years, with prior infarct in 23 per cent of cases, and an in-hospital all-cause mortality rate of 22 per cent) - these individuals constituted the group B; 2 ) a study with 764 AMI patients, consecutively admitted within the first three hours of STEMI during the 1982-1989 period (with a mean age of 59 years, with prior infarct in 24 per cent of cases, and a 30-day mortality rate of eight per cent in patients who did not experience clinically significant brady- or tachyarrhthmias that is, rhythm disturbances implying a therapeutical intervention or a modification of the clinical monitoring) - these individuals constituted the group C.

The study was approved by the local ethics committee and was in accordance with the 2008 Declaration of Helsinki.

\section{Statistical Analysis}

The results are presented as means (and standard deviations) and percentages. The chi-square method (with the Yates correction) and the exact Fisher test were used for comparison of categorical variables. Student's $t$ test was used for comparison of numerical variables. A p-value of less than 0.05 was considered as statistically significant.

\section{Results}

\section{General data}

The patients in group A presented with the following prevalences of preexistent, indetermined and acutely acquired IVB: 14.5 per cent, 1.0 per cent and 27.6 per cent, respectively. According to the different types of acute IVB considered, their prevalences were as follows: isolated LAH - 15.0 per cent globally, and specifically 1.8 per cent for advanced LAH; isolated $\mathrm{LPH}-1.3$ per cent; isolated (incomplete or complete) RBBB - 3.3 per cent; RBBB associated to LAH - 3.3 per cent; RBBB associated to LPH - 0.2 per cent; complete LBBB - 2.1 per cent; aspecific left IVB - 2.3 per cent; aspecific bilateral IVB - 0.7 per cent.

In 52 per cent of cases, acquired IVB was transient. Despite this and the fact that an acute IVB occurred in one third of patients with preexistent IVB, the final IVB prevalence in survivors (at discharge) was 24.4 per cent. This means that the survivors showed an increase of at least 57 per cent of the electrocardiographic IVB prevalence in comparison with what the global population exhibited prior to STEMI or at the admission ECG recording.

In patients with transient acquired IVB, the temporary feature of the conduction defect did not directly correlate with the CA chronology: the coronary intervention was performed $403+-372$ minutes after the STEMI initiation in patients with transient IVB, and $300+-192$ minutes after the AMI start in individuals with sustained IVB (these figures refer to the time interval elapsed between the AMI initiation and the moment when the AMI-related artery was effectively re-opened).

In eight per cent of transient IVB cases, the conduction defect was markedly sporadic, vanishing spontaneously before the coronary intervention. However, in 71 per cent of cases, the IVB disappearance seemed to be CA-correlated: in most cases, the return of the intraventricular conduction to the pre-STEMI pattern occurred immediately or almost immediately after the coronary intervention.

On the other hand, in 21 per cent of patients with transient IVB, the conduction disturbance occurred after the coronary angioplasty. Interestingly, in these individuals, a persistent no-reflow phenomenon or the lack of CA success were registered in only a minority of cases (11 per cent, a percentage only slightly higher than the figure of 8.6 per cent presented by the global population). 
More interestingly, most of the sustained cases of acquired IVB (60 per cent) occurred after the coronary catheterisation. Under these circumstances, the combined evidence of unsuccessful CA, persistent no-reflow or exclusion of coronary intervention was again recorded in only a minority of patients (15 per cent).

Patients with preexistent or in determined IVB (with a mean age of $70+-12$ years) and patients with acquired IVB (with a mean age of $65+-14$ years $)$ were significantly older $(\mathrm{p}<0.001)$ than the individuals that did not show any evidence of IVB prior to or throughout the STEMI acute phase (mean age of $60+-13$ years). On the other hand, among patients with acquired IVB, the individuals showing RBBB or complete LBBB were noticed by their advanced age (means of $68+-15$ years and $68+-12$ years, respectively).

Comparing with IVB-free individuals, patients with stable preexistent or in determined IVB had a higher in-hospital mortality (11.1 per cent vs 5.1 per cent; $\mathrm{p}>0.1$ ), being clearly old the great majority of the deceased subjects (age over 75 years), in contrast with the deceased IVB-free patients. On the other hand, patients with acquired intraventricular conduction defects had a significantly higher in-hospital mortality than IVB-free subjects (13.2 per cent vs 5.1 per cent; $\mathrm{p}<0.01)$.

[Table 1] shows the mortality rates in patients with acquired IVB, discriminated according to the IVB type. Individuals with acute major IVB, with a global prevalence of 12.9 per cent, had an overall mortality of 26.9 per cent, and their fatal cases accounted for 95 per cent of the total mortality registered in the acquired IVB subgroup. Nevertheless, that high mortality was mostly concentrated in patients with RBBB (isolated or associated with LAH) and complete LBBB.

As long as the in-hospital mortality of patients with acquired major IVB is concerned, it is also important to distinguish between transient and sustained ('permanent') forms of major IVB: the mortality in patients showing a transient IVB (incorporating, in cases of RBBB associated with left IVB, only the transient feature concerning the right-sided IVB, independently of being the left-sided IVB component transient or not) was 19.3 per cent, contrasting with 47.6 per cent for patients presenting with sustained IVB $(\mathrm{p}<0.05)$.

\section{Pre-existent intraventricular block}

Among patients surely presenting with a preexistent IVB, a fraction composed of 58 individuals did not exhibit any kind of new (acquired) intraventricular conduction disturbance, namely the aggravation of the prior IVB pattern. The clinical profile of these patients displayed a mean

Table 1. In-hospital mortality in patients with AMI complicated by acquired intraventricular block

\begin{tabular}{|c|c|}
\hline IVB type & Mortality \\
\hline Isolated LAH (global value) * & $1.1 \%$ \\
\hline Isolated de novo $\mathrm{LAH}$ & $0 \%$ \\
\hline Advanced LAH & $0 \%$ \\
\hline Isolated LPH & $1.5 \%$ \\
\hline Isolated RBBB & $3.0 \%$ \\
\hline RBBB associated with LAH & $45.0 \%$ \\
\hline RBBB associated with LPH & $0 \%$ \\
\hline Complete LBBB & $35.7 \%$ \\
\hline Aspecific left IVB & $7.1 \%$ \\
\hline \multicolumn{2}{|c|}{$\begin{array}{l}\text { Abbreviations: AMI - acute myocardial infarction; IVB - intraventricular block; LAH } \\
\text { - left anterior hemiblock; LPH - left posterior hemiblock; RBBB - right bundle branch } \\
\text { block; LBBB - left bundle branch block. } \\
\text { Annottations: ( }{ }^{*} \text { ) englobing cases with preexistent LAH but aggravated during AMI } \\
\text { course. }\end{array}$} \\
\hline
\end{tabular}

age of $69+-12$ years, and prevalence's of 17 per cent for female gender, 46.6 per cent for diabetes, 41.4 per cent for chronic renal disease, 8.6 per cent for multiple territory vascular disease, 43.1 per cent for anterior wall AMI, 44.8 per cent for large STEMI, and 17.2 per cent for severe systolic left ventricular dysfunction (ejection fraction $<35$ per cent). All but one underwent primary CA $370+-293$ minutes after the initiation of symptoms, and the combined endpoint of unsuccessful CA or persistent post-CA no-reflow was recorded in 13.8 per cent of individuals. An extremely severe lesion of the AMI culprit artery (considered as: a) an occlusive or sub-occlusive lesion located in the left main coronary [LMC] artery, the ostium of the left anterior descending [LAD] artery, or the ostium of the circumflex artery; b) an occlusive proximal lesion of LAD artery or circumflex artery; c) an occlusive/ sub-occlusive ostial lesion or an occlusive proximal lesion of the right coronary artery [RCA], in case of right dominance) was found in 29.3 per cent of patients.

The in-hospital mortality rate was 6.9 per cent in this subgroup; the mean age of the deceased patients was $76+-4$ years.

\section{Acquired left bundle branch block}

In our 2008-2012 population, we found 14 cases of acquired complete LBBB, with a QRS duration between 120 and $170 \mathrm{msec}$; the QRS axis was normal in 13 subjects and a left axis deviation was evident in one individual (with prior LAH). Eight patients (62 per cent) had prior evidence of IVB (left IVB in seven, and RBBB in one).

Acquired LBBB initially coursed with associated AV block in four patients (complete AV block in three, and first-degree AV block in one). The AV conduction disturbance was transient, vanishing immediately after the coronary intervention, in all individuals.

LBBB was clearly a phase- 4 brady dependent conduction defect (always associated with concomitant complete AV block) in two patients and was tachy dependent in another. Death occurred in the tachy dependent LBBB patient and in one of the brady dependent LBBB cases. A patient with advanced LAH alternating with LBBB did not display any LBBB tachy dependence.

LBBB was transient in 85 per cent of cases $(n=11)$, usually disappearing $(\mathrm{n}=10)$ after the coronary intervention, either immediately $(n=6)$ or after a period of several hours $(n=2)$ or days $(n=2)$. In one case, a partial post-CA IVB regression was seen, persisting a permanent aspecific IVB pattern at discharge. In another case, a post-CA acquired intermittent $\mathrm{LBBB}$ was observed, later receding to advanced $\mathrm{LAH}$, that persisted stably until death occurred. The lack of immediate full vanishment after the coronary intervention constituted an ominous sign in patients showing early-acquired LBBB during the STEMI acute phase: three out of six individuals died.

Two patients showed lately-acquired permanent LBBB: the LBBB pattern occurred after the coronary angioplasty in individuals with chronic pre-infarction advanced left IVB; there was a conduction defect aggravation during the AMI course, but these patients turned to be AMI survivors after all.

Only 64 per cent $(n=9)$ of patients with acquired LBBB had an early admission (within the first three hours of STEMI evolution), and this was reflected on the admission mean time ( $257+-459$ minutes). CA was equally performed with some delay: the eleven patients undergoing primary $\mathrm{CA}$ (the remaining subjects were submitted to rescue CA) saw the coronary intervention being performed $607+-523$ minutes after the AMI initiation and $307+-301$ minutes after the first 
medical attendance. Only five individuals (36 per cent) had a coronary intervention performed less than two hours after the admission, thus reflecting how difficult were the making of a STEMI diagnosis in the setting of AMI complicated by LBBB; it is noteworthy that survival occurred in all but one of these patients (half of them with an ejection fraction less than 35 per cent). Only two patients (14 per cent) with acquired LBBB underwent primary CA within the first three hours of AMI evolution; both survived.

Seventy-nine per cent $(n=11)$ of patients with acquired LBBB had a large STEMI. In the whole subgroup, AMI was located on the anterior wall in six individuals, on the inferior wall in six (being biventricular in two), and the infarction site was combined (anterior plus inferior walls) in the remaining two patients.

Eighty-six per cent $(n=12)$ of patients had severe or very severe coronary artery disease: the angiographic characteristics are depicted in [Table 2]. An extremely severe lesion of the AMI culprit artery (considered as: a) occlusive/sub-occlusive lesion of LMC artery or the ostium of LAD artery or the ostium of the circumflex artery; b) proximal occlusion of LAD artery or the circumflex artery; c) ostial or proximal RCA occlusion/sub-occlusion, in case of right dominance; d) occlusion of a non-distal bypass graft to the culprit artery) was found in 71 per cent of patients $(n=10)$ : LAD artery was the AMI culprit vessel in seven individuals, RCA in five, the circumflex artery in one, and in another patient the AMI responsibility was 'shared' by a double lesion (occluded bypass grafts to LAD artery and RCA).

Every patient showed systolic left ventricular dysfunction, which was severe (ejection fraction $<35$ per cent) in nine individuals ( 64 per cent).

The occurrence of an unsuccessful CA or a persistent no-reflow phenomenon was seen in three patients (21.4 per cent); death occurred in two. Curiously, the acquired LBBB was transient in two cases, and in third one, LBBB partially receded to a stable advanced LAH pattern.

The prevalence of diabetes was slightly higher than the one seen in the general LBBB- or RBBB-free STEMI population (50 per cent vs 36 per cent; $p>0.25$ ), and the presence of other significant comorbidities (chronic renal disease, multiple territory vascular disease, chronic obstructive pulmonary disease or obesity) was remarkable (involving 71 per cent of patients), and the highlight is addressed to chronic kidney disease (evident in half of the patients). On the other hand, the mean age of the deceased patients was clearly higher than the mean age of survivors ( $75+-8$ years vs $65+-13$ years, respectively; $p>0.1$ ).

\section{Acquired right bundle branch block}

The group A comprised 43 patients (7.1 per cent of total) with acquired RBBB, occurring de novo in 40 individuals, and showing the aggravation of a prior right IVB in three. An aggravated or de novo

Table 2. Angiographic characteristics of the coronary disease in patients with complete LBBB

\begin{tabular}{|l|l|}
\hline 3-vessel disease & $\begin{array}{l}\text { 6 patients (3 with severe lesion of LMC artery; 5/6 patients with } \\
\text { inferior wall AMI) }\end{array}$ \\
\hline 2-vessel disease & 6 patients \\
\hline LAD + Cx & 3 patients \\
\hline LAD + RCA & 2 patiens \\
\hline RCA + Cx & 1 patient (with preexistent RBBB + LPH) \\
\hline 1-vessel disease & 1 patient (LAD artery ostial occlusion) \\
\hline $\begin{array}{l}\text { Abbreviations: LBBB - left bundle branch block; LMC - left main coronary; AMI } \\
\text { - acute myocardial infarction; LAD - left anterior descending; Cx - circumflex; } \\
\text { RCA - right coronary artery; RBBB - right bundle branch block; LPH - left posterior } \\
\text { hemiblock. }\end{array}$ \\
\hline
\end{tabular}

RBBB appeared as an isolated IVB event in 20 patients; in four out of these individuals, RBBB was associated with a prior left IVB (stable along the STEMI course), but in other four patients, although an acquired left IVB did also occur, its appearance was dissociated from the RBBB depiction and manifested either before or after RBBB became clear. In the remaining 23 patients, who showed bilateral acquired IVB, the biventricular conduction disturbances occurred simultaneously or almost simultaneously: LAH was the left IVB more often seen in combination with $\mathrm{RBBB}(\mathrm{n}=20)$, but there was one instance with an association with LPH and two cases of aspecific left IVB combined with the right-sided conduction defect.

Either in cases of isolated RBBB or in instances with concomitantly acquired left IVB, the right conduction defect could present a transient or sustained course. A sustained RBBB was associated with higher mortality, either in isolated RBBB ( 40 per cent vs 20 per cent for transient defects; $\mathrm{p}=0.244$ ) or in RBBB associated with LAH (75 per cent vs 25 per cent for patients with transient RBBB; $\mathrm{p}=0.037$ ).

Looking now at the instances of left IVB acquired simultaneously or almost simultaneously with RBBB, the behaviour of the left conduction defect could also either transient $(n=12)$ or sustained $(\mathrm{n}=11)$, accompanying or not the RBBB course. In the much more frequent condition of $\mathrm{RBBB}$ associated with $\mathrm{LAH}$, the combination of transient RBBB with transient LAH was twice as frequent as the combination of transient RBBB with sustained LAH; on the other hand, the combination of sustained RBBB with sustained LAH was three times as frequent as the combination of sustained RBBB with transient $\mathrm{LAH}$. Thus, when RBBB combined with LAH occurred de novo in the clinical setting of STEMI, the LAH behaviour through the AMI course showed a clear trend to be in syntony with the ECG behaviour of the right-sided IVB.

The behaviour of the left component of acquired bilateral IVB throughout the STEMI acute phase did not seem to be related with the mortality risk. The in-hospital mortality was only associated with the transient or sustained character of the right-sided intraventricular conduction disturbance.

Complete or high-degree AV block was associated with acquired RBBB in five patients (prevalence of 11.6 per cent), always in the setting of RBBB combined with left IVB: this happened in three cases of RBBB plus LAH (prevalence of 15 per cent in this acquired bilateral IVB), in one instance with advanced aspecific left IVB, and in one patient with preexistent LBBB. Four of these individuals, all aged over 78 years, needed temporary pacing: the AV conduction did not improve following the coronary catheterisation procedure, and three patients remained pacing-dependent until death occurred. In this subgroup of patients showing AV block (in whom the coronary intervention took place $389+-328$ minutes after the AMI initiation, with a relative unsuccess - two patients exhibited persistent partial no-reflow), the in-hospital mortality was very high, of 80 per cent (corresponding to four deaths).

Only 16.3 per cent of patients with acquired RBBB did not have an early hospital admission; they were admitted more than three hours after the AMI initiation. In patients undergoing CA $(n=38)$, the coronary intervention was performed $286+-230$ minutes following the beginning of the coronary event; this figure grossly corresponds to the intervention profile registered in the general population, but clearly contrasts with what was recorded in patients with acquired LBBB (in these subjects, the 'late' admission rate was 36 per cent, and the coronary procedure was performed with a mean delay of more than 300 minutes in comparison with the acquired-RBBB patients undergoing coronary 
intervention). On the other hand, 35 per cent of the patients underwent CA up to three hours of AMI evolution, but about half of them did not survive (seven out of 15 subjects); however, the fact that four of five patients submitted to a coronary intervention within the first two hours of STEMI evolution did survive, deserves to be highlighted.

Seventy-three per cent of patients with acquired RBBB had a large STEMI. An anterior wall STEMI was present in 78 per cent of subjects, and the remaining individuals had an inferior wall AMI (usually not large). Seventy-eight per cent of patients showed systolic left ventricular dysfunction, which was severe (ejection fraction less 35 per cent) in only 40 per cent of cases $(n=16)$.

An unsuccessful CA or a persistent no-reflow post-CA angiographic pattern were seen in 18.6 per cent of patients with acquired RBBB, and these post-procedure complications were significantly more frequent than in RBBB-free patients ( 7.8 per cent; $\mathrm{p}<005)$.

The diabetes prevalence was 56 per cent, significantly higher than in RBBB-free patients ( 36 per cent; $\mathrm{p}<0.025$ ). On the other hand, the presence of other significant comorbidities (chronic renal failure, multiple territory vascular disease, chronic obstructive pulmonary disease or obesity) was important (63 per cent of patients), being relevant the role of chronic kidney disease (diagnosed in 42 per cent of individuals).

Among the patients with acquired RBBB, the deceased subjects had a mean age of $76+-12$ years, significantly higher than the mean age of survivors $(63+-14$ years; $p<0.01)$. Seventy-five per cent of patients older than 78 years did not survive.

Comparing clinical characteristics from patients displaying acutely acquired RBBB with those presented by their respective matched controls [Table 3], we can notice that, if the comorbidities

Table 3. Clinical characterization of patients with acquired RBBB and respective matched controls

\begin{tabular}{|c|c|c|c|}
\hline & $R B B B$ & Controls & $p$ \\
\hline Age (years; mean $\pm \mathrm{SD}$ ) & $68 \pm 15$ & $68 \pm 10$ & - \\
\hline Female gender (n) & 10 & 10 & - \\
\hline Large STEMI & $67.5 \%$ & $67.5 \%$ & - \\
\hline Prior infarct & $12.5 \%$ & $17.5 \%$ & $>0.75$ \\
\hline Diabetes mellitus & $57.5 \%$ & $57.5 \%$ & - \\
\hline percentage of patients under treatment & $39 \%$ & $61 \%$ & 0.081 \\
\hline Other significant comorbidities * & $65.0 \%$ & $65.0 \%$ & - \\
\hline \multicolumn{4}{|l|}{ AMI culprit artery } \\
\hline LMC / LAD (n) & 34 & 34 & - \\
\hline $\mathrm{RCA} / \mathrm{Cx}(\mathrm{n})$ & 6 & 6 & - \\
\hline \multicolumn{4}{|l|}{ Coronary angiographic data } \\
\hline 3-VD / LMC & $42.5 \%$ & $37.5 \%$ & $>0.75$ \\
\hline 2-VD & $22.5 \%$ & $22.5 \%$ & - \\
\hline 1-VD (LAD) & $30.0 \%$ & $32.5 \%$ & 0.975 \\
\hline Left ventricular dysfunction $(\mathrm{EF}<55 \%)$ & $70.0 \%$ & $77.5 \%$ & $>0.5$ \\
\hline $45-54 \%$ & $12.5 \%$ & $32.5 \%$ & $>0.05$ \\
\hline $35-44 \%$ & $17.5 \%$ & $32.5 \%$ & $>0.1$ \\
\hline$<35 \%$ & $40.0 \%$ & $12.5 \%$ & $<0.025$ \\
\hline $\begin{array}{l}\text { Admission time interval (minutes) } \\
\text { [patients undergoing primary CA] }\end{array}$ & $151 \pm 176$ & $191 \pm 193$ & $>0.3$ \\
\hline \multicolumn{4}{|c|}{$\begin{array}{l}\text { Abbreviations: RBBB - right bundle branch block; SD - standard deviation; STEMI - } \\
\text { ST elevation myocardial infarction; AMI - acute myocardial infarction; LMC - left main } \\
\text { coronary artery; LAD - left anterior descending artery; RCA - right coronary artery; } \\
\text { Cx - circumflex artery; VD - vessel disease; EF - ejection fraction; CA - coronary } \\
\text { angioplasty. } \\
\text { Annotations: (") presence of chronic renal disease, multiple territory vascular disease, } \\
\text { chronic obstructive pulmonary disease / obesity. }\end{array}$} \\
\hline
\end{tabular}

are concerned, the diabetic acquired-RBBB patients (although having an identical prevalence of the metabolic disease) exhibited a higher clinical risk than the control subjects, since they showed a clear trend to be less often treated with chronic anti-diabetic therapy. On the other hand, whether the general characteristics of coronary artery disease, the chest pain-admission time intervals and the prevalence of systolic left ventricular dysfunction did not differ between the two subgroups, the presence of severe systolic dysfunction (ejection fraction $<35$ per cent) was significantly more frequent in acquired-RBBB patients ( $\mathrm{p}$ $<0.025$ ). Additionally, in cases of anterior wall STEMI, a very severe lesion of the AMI culprit artery (an occlusive/sub-occlusive lesion of LMC artery or LAD artery ostium, or a proximal LAD artery occlusion) was significantly more frequent in RBBB patients than in matched controls (50 per cent vs 22.5 per cent; $\mathrm{p}<0.025$ ). Further, RBBBpatients in-hospital mortality ( 37.5 per cent) was significantly $(\mathrm{p}<$ 0.025 ) higher than the one recorded in their matched controls (5.0 per cent), which was not different from the value shown by the global IVBfree population. Furthermore, considering the patients undergoing primary $\mathrm{CA}$, an unfavorable clinical evolution was not necessarily associated with a 'late' coronary intervention: the 'chest pain-balloon' time intervals were not significantly different either in survivors (309 +- 260 minutes in RBBB patient's vs $335+-220$ minutes in controls; NS) or in deceased individuals (277 +- 210 minutes in RBBB subject's vs 90-600 minutes in the two dying control patients; NS).

\section{Comparison with the pre-thrombolytic era}

By comparing patients from the Group A (2008-2012 period) and Group B (1980-1982 interval), we can observe that: 1) the in-hospital mortality recorded in patients with acquired LBBB was moderately higher in the $1980-1982$ period ( 46.7 per cent vs 35.7 per cent in the 2008-2012 period), but the 24 per cent relative risk reduction observed nowadays is not statistically significant $(\mathrm{p}=0.248) ; 2)$ the in-hospital mortality in patients with acquired or indetermined RBBB was clearly higher in the 1980-1982 period (58 per cent vs 37 per cent in the 20082012 interval),but again this 36 per cent relative risk reduction, mainly attributable to the introduction of primary CA, cannot be considered as statistically significant $(\mathrm{p}>0.1)$.

Comparing the in-hospital all-cause mortality registered in patients with acquired advanced IVB from the Group A and the Group C (1982-1989 period), and having been used the same IVB definitions and the same ECG diagnostic criteria in both Groups, one can observe a very significant reduction of the mortality risk $(p<0.001)$ from the pre-thrombolytic era (1982-1989) to the present era, characterized by the use of primary CA (from 62 per cent to 27 per cent, a 56 per cent relative risk reduction).

\section{Discussion}

In the present era, an acutely acquired IVB remains a relatively frequent complication during the STEMI acute phase. A serial ECG screening throughout the first days of AMI evolution, even if continuous recordings are used, does not allow the depiction of all manifestations of intraventricular conduction disturbances, namely an acutely acquired LAH or LPH. A minority of LAH or LPH cases may be masked by opposite axial deviations, determined by lateral wall infarction (in case of acquired LAH) or inferior wall necrosis (in case of acquired LPH); in this context, masked fascicular blocks can only be fully diagnosed by means of vectorcardiography [11]. On the other hand, in case of concordant causes of left axis deviation - for instance, before the association of inferior wall infarction with LAH - serial 
electro-vectorcardiography studies allowed to build adequate ECG diagnostic criteria for LAH detection [9].

In spite of the referred diagnostic limitations, a serial conventional ECG evaluation allows to obtain a quite fair and clinically useful idea about the prevalence of acquired IVB in the setting of patients presenting with STEMI. Thus, the comparison of the results obtained by our Group in the present era with those collected twenty-three and thirty years previously ( in the pre-thrombolytic era, through serial electrocardiographic [10] and electro-vectorcardiographic studies $[2,12]$, respectively) - and it must be said that in those previous studies were used ECG diagnostic methodologies similar to the ones utilized in our present study - suggests that the present prevalence of acquired IVB during the STEMI acute phase is reasonably superposable to the prevalences seen in the past.

The maintenance of a relatively high prevalence of acute IVB, in the era when the emergent mechanical coronary reperfusion ascended to a very relevant position in the ranking of the AMI therapeutical armamentarium, is a consequence of these conduction defects being manifested mostly in the first hours and even the first minutes of the AMI clinical evolution, often before the implementation of the reperfusion treatment.

The 'spontaneous' variation [13] of an acquired IVB or its full regression [1] along the STEMI acute phase were well known in the pre-thrombolytic era. The implementation of an effective reperfusion therapy - as primary CA is - in the first hours of STEMI evolution allowed the transitoriness of an intraventricular conduction defect to become much more frequent nowadays [5].

Nevertheless, in Group A, about one fifth of transient cases and more than half of sustained acquired IVB cases occurred following the coronary intervention, and that appearance was not consistently related to a fully or partially unsuccessful mechanical reperfusion. The best explanation points to the combination of transient ischemia (eventually durable) of a certain segment of the intraventricular conduction system with prior degenerative lesions ('silent' from an ECG viewpoint). The 'visible' ECG consequences of the ischemic insult would be immediately facilitated by previous (variable) functional conduction disturbances affecting many fiber bundles in the area of the conduction system presenting the chronic degenerative disease: on one hand, ischemia would functionally inactivate previously normofunctioning islets of the conduction tissue; on the other hand, ischemia would provoke the transient, prolonged or permanent aggravation of the conduction disturbances in previously dysfunction areas. Consequently, the functionally affected zone of the intraventricular conduction network would become larger, determining (after a latent period) a frank modification of the intraventricular conduction pattern, enough to produce an IVB electrocardiographic picture (transient or sustained). This explanation is also consistent with the fact that one third of preexistent IVB patients (from Group A) have shown a new IVB type (joining the prior conduction defect) or a prior IVB aggravation.

STEMI patients with prior IVB were clearly more aged than individuals that were IVB-free now of AMI initiation. Patients presenting with acquired complete LBBB or de novo RBBB (incomplete or complete) had also an advanced mean age. On the other hand, fourty per cent (17 out of 43 ) of subjects showing acquired RBBB provided ECG features suggestive of the presence of degenerative disease of the intraventricular conduction system prior to AMI occurrence. Combining these facts with some data referring to cases with post-CA acquired IVB, we can suggest that it is likely that in more than half (53 per cent) of patients with post-AMI acquired IVB, the occurring conduction defect will be, at least partially, the result of the interaction of ischemic lesions with chronic degenerative lesions of the conduction system.

The data analysis from three different cohorts of patients - patients with prior IVB who did not present with de novo IVB, the subgroup of IVB-free individuals, and the subjects from the control subgroup matched with acquired-RBBB patients - we are able to state that the presence of a prior IVB, being stable during the STEMI acute phase, does not constitute a relevant prognostic factor. The slight increase in mortality seen in these patients, when compared with IVB-free individuals (6.9 per cent vs 5.1 per cent), is attributed to a more advanced age and higher prevalence's of diabetes and severe systolic left ventricular dysfunction.

Occurrence of acquired LBBB during the STEMI acute phase usually means that we are before an aged patient, frequently with chronic degenerative disease of the conduction system, and presenting with severe or critical coronary artery disease. In this context, the acute coronary syndrome will show a severe clinical picture and will determine an extensive and severe ischemia of the anterior and posterior fascicles of the distributive network of the so-called left bundle branch; the functional effect of ischemia is eventually added to the one provoked by prior degenerative lesions of the left conduction system. In this setting, LBBB electrocardiographic pattern is a mere but excellent early marker of bad prognosis, independently of being transient or not. The bad prognosis shown by many patients is the result of a very severe STEMI clinical picture associated with advanced age and frequent significant comorbidities. A delay in hospital admission and the difficulties frequently posed, at the admission room, to the ECG diagnosis of STEMI in presence of complete LBBB often contribute to a significant delay in performing an emergent coronary intervention: this delay does not facilitate an attempt to modify decisively the prognosis by means of a successful coronary intervention.

It is noteworthy that the immediate post-CA regression of LBBB does not necessarily mean a better clinical prognosis. Finally, our data suggest - and this must be emphasized - that STEMI patients with acquired complete LBBB need an early coronary intervention with effective reperfusion achieved within the first two or three hours of AMI evolution.

Occurrence of acquired RBBB during STEMI usually means that we are before an aged patient with a large anterior wall STEMI, frequently diabetic and presenting with other significant comorbidities, and frequently exhibiting a critical lesion in the LAD artery territory (which not rarely predicts a troubled procedure whenever an effective CA is concerned). A sustained RBBB pattern (especially when associated to acquired LAH) and age over 75 years constitute markers of bad prognosis, and in this setting the in-hospital mortality is very high despite the coronary intervention. An early primary CA, performed within the first two hours of AMI evolution, is probably mandatory if one can revert the initial prognostic evaluation.

In the era of mechanical reperfusion as the gold standard for STEMI treatment, emergent CA allowed to significantly reduce the mortality in patients presenting with STEMI complicated by advanced IVB. However, whether the coronary intervention allowed to abolish the excess of mortality once shown by patients with several types of advanced IVB, this does not apparently happen nowadays when the acquired IVB type complicating STEMI is LBBB or RBBB: in these 
patients, the relatively modest reduction seen in in-hospital mortality seems to reflect more the global improvement in efficacy of AMI intensive therapy than a decisive result from the coronary intervention.

The practice of emergent coronary reperfusion in STEMI patients is characterised, in the 'real world', by a coronary intervention that is not often performed very early. Patients with STEMI complicated by acquired LBBB or RBBB apparently need a coronary intervention performed within the first two hours of AMI evolution, to allow their prognosis to be very favourably affected, although the clinical evolution in these severely ill patients is influenced by many variables.

\section{Conclusions}

In the era of mechanical reperfusion of ST elevation myocardial infarction, applied in the 'real world', the presence of a preexistent intraventricular block does not influence the prognosis, but an acquired intraventricular conduction defect still implies a reserved prognosis under specific circumstances. In general, an emergent coronary angioplasty significantly improved the prognosis of several types of advanced intraventricular conduction defects acquired during acute myocardial infarction but did not globally and significantly modify the bad prognosis shown by patients presenting with ST elevation myocardial infarction complicated by sustained complete left bundle branch block or sustained right bundle branch block (either isolated or associated with left anterior hemiblock). The bad prognosis of these patients seems to improve notoriously whenever the coronary intervention is performed within the first two hours of myocardial infarction evolution. However, to get most patients with ST elevation myocardial infarction, complicated by acutely acquired left bundle branch block, undergoing early primary coronary angioplasty still constitute a clinical challenge in our days.

\section{References}

1. Lie KI, Wellens HJ, Schuilenburg RM (1976) Bundle branch block and acute myocardial infarction. In: Wellens HJJ, Lie KI, Janse MJ, editors. The conduction system of the heart. Structure, function and clinical implications. Leiden: HE Stenfert Kroese BV p:662-672.
2. Bordalo ADB, Bordalo e Sá AL, Ferreira RJ, Ribeiro C (1983) Clinical and electro cardiologic features of acquired intraventricular blocks in acute myocardial infarction. In: Lotto A, editor. Recenti acquisizioni nella diagnose e terapia delle aritmie cardiache (Giornate Internazionali - Abstracts Book). Milano: Istituto di Ricovero e Cura à Carattere Scientifico (Ospedale Maggiore di Milano) p:33-37.

3. Moreno AM, Alberola AG, Tomás JG, Chávarri MV, Soria FC, et al. (1997) Incidence and prognostic significance of right bundle branch block in patients with acute myocardial infarction receiving thrombolytic therapy. Int J Cardiol 61: 135-141. [Crossref]

4. Wong CK, Stewart RA, Gao W, French JK, Raffel C et al. (2006) Prognostic differences between types of bundle branch block during the early phase of acute myocardial infarction: insights from the Hirulog and Early Reperfusion or Occlusion (HERO)-2 trial. Eur Heart J 27: 21-28. [Crossref]

5. Tomoda H, Aoki N (2005) Right bundle branch block in acute myocardial infarction treated by primary coronary angioplasty and stenting. Angiology 56: 131-136. [Crossref]

6. Kurisu S, Inoue I, Kawagoe T, Ishihara M, Shimatani Y, et al. (2007) Right bundlebranch block in anterior acute myocardial infarction in the coronary intervention era: acute angiographic findings and prognosis. Int J Cardiol 16: 57-61. [Crossref]

7. Widimsky P, Rohác F, Stásek J, Kala P, Rokyta R et al. (2012) Primary angioplasty in acute myocardial infarction with right bundle branch block: should new onset right bundle branch block be added to future guidelines as an indication for reperfusion therapy? Eur Heart J 33: 86-95. [Crossref]

8. Bayés de Luna A (2012) Ventricular blocks. In: Bayés de Luna A, editor. Clinical Electrocardiography, $4^{\text {th }}$ ed. Chichester: Wiley-Blackwell p:158-202.

9. Longo A, Bordalo ADB, Ribeiro C (1981) Re-evaluation of electrocardiographic criteria for diagnosis of left anterior segmental block associated with inferior infarction. Arch Mal Coeur Vaiss 74: 179-184.

10. Bordalo ADB, Varela MG, Almeida MA, Bordalo-Sá AL, Ferreira D et al. (1993) Early myocardial infarction arrhythmias. In: Lopo Tuna J, editor. Cardiac Arrhythmias: Clinical aspects. Lisboa: Permanyer Portugal p: 239-245.

11. Bordalo ADB, Lacximy I, Ribeiro C (1978) Diagnosis of left fascicular blocks masked by myocardial infarction. Adv Cardiol 21: 249-252. [Crossref]

12. Bordalo ADB, Lacximy I, Correia R, Ribeiro C (1977) Vectocardiographic analysis of left-sided QRS in acute myocardial infarction (Serial study of two hundred cases). $J$ Médico 94: 269-276.

13. Ribeiro C, Bordalo ADB, Lacximy I (1978) Unstable axis deviation during the acute phase of myocardial infarction. Serial vectorcardiographic study of 200 cases. $A d v$ Cardiol 21: 253-258. [Crossref]

Copyright: (C2018 Bordalo ADB. This is an open-access article distributed under the terms of the Creative Commons Attribution License, which permits unrestricted use, distribution, and reproduction in any medium, provided the original author and source are credited. 